\title{
Commentary
}

\section{Recommendations to Optimize the Safety of Elective Surgical Care While Limiting the Spread of COVID-19: Primum Non Nocere}

\author{
Ron Gilat, M.D., Eric D. Haunschild, B.S., Tracy Tauro, B.S., B.A., and \\ Brian J. Cole, M.D., M.B.A.
}

\begin{abstract}
COVID-19 has drastically altered our lives in an unprecedented manner, shuttering industries and leaving most of the country in isolation as we adapt to the evolving crisis. Orthopedic surgery has not been spared from these effects, with the postponement of elective procedures in an attempt to mitigate disease transmission and preserve hospital resources as the pandemic continues to expand. During these turbulent times, it is crucial to understand that although patients' and care-providers' safety is paramount, canceling or postponing essential surgical care is not without consequences and may be irreversibly detrimental to patients' health and quality of life in some cases. The optimal solution to how to balance effectively the resumption of standard surgical care while doing everything possible to limit the spread of COVID-19 is undetermined and could include such strategies as social distancing, screening forms and tests, including temperature screening, segregation of inpatient and outpatient teams, proper use of protective gear, and the use of ambulatory surgery centers (ASCs) to provide elective, yet ultimately essential, surgical care while conserving resources and protecting the health of patients and health care providers. Of importance, these recommendations do not and should not supersede evolving United States Centers for Disease Control and Prevention and relevant federal, state and local public health guidelines. Level of Evidence: Level V.
\end{abstract}

$\mathbf{T}$ he first confirmed case of COVID-19 in the United States was reported in northern Washington on January 20 of this year. Since then, the 2019 Novel Coronavirus (COVID-19) has upended our society and placed an unprecedented strain on health care systems across the country. ${ }^{1}$ Orthopedic surgery has not been

From Midwest Orthopaedics at Rush University Medical Center, Chicago, Illinois, U.S.A. (R.G., E.D.H., T.T., B.J.C.); and Department of Orthopaedic Surgery, Shamir Medical Center and Tel Aviv University, Tel Aviv, Israel (R.G.).

The authors report the following potential conflict of interest or source of funding: This paper has received the Orthoregeneration Network (ON) Foundation Literature Grant (of 1500CHF). B.J.C. reports IP royalties, consultant fees, and research support from Arthrex; research support from Aesculap, outside the submitted work; other financial or material support from Athletico, outside the submitted work; and IP royalties from Elsevier. Full ICMJE author disclosure forms are available for this article online, as supplementary material.

Received April 18, 2020; accepted April 20, 2020.

Address correspondence to Brian J. Cole, M.D., M.B.A., 1611 W Harrison St., Chicago, IL 60612,U.S.A. E-mail: brian.cole@rushortho.com

(C) 2020 THE AUTHORS. Published by Elsevier Inc. on behalf of the Arthroscopy Association of North America. This is an open access article under the CC BY-NC-ND license (http://creativecommons.org/licenses/by-nc-nd/4.0/).

2666-061X/20631

https://doi.org/10.1016/j.asmr.2020.04.008 spared from these drastic changes, particularly when considering the widespread cessation of elective clinical and surgical care. Most of these elective procedures have been postponed in the interest of patient and provider health and to address anticipated shortages in staffing, beds, ventilators, and personal protective equipment (PPE) as the virus rapidly spreads through our communities. ${ }^{2}$ This mandate effectively halted the traditional clinical structure of orthopedic practices, necessitating a new system for providing the highest quality of orthopedic care while practicing the principles of social distancing and preventive measures to avoid the transmission of COVID-19. Although a shift to telemedicine has proven instrumental in providing care, questions about what is defined as an essential procedure have come to the forefront. A preeminent concern in this conversation is how delays in care, resulting in daily pain, functional disability and unbearable financial damages will affect the long-term physical and mental health, employment capacity and overall well-being of patients and of our economy. Experiences in Hong Kong and Singapore have shown that mitigation strategies, such as social distancing, temperature screenings, inpatient/outpatient teams, and the use of ambulatory surgery centers (ASCs), have 
been effective in providing essential surgical care while conserving resources and protecting the health of patients and health care providers. ${ }^{3}$

The purpose of this investigation was to present and analyze the most up-to-date evidence available concerning how the COVID-19 pandemic has affected the orthopedic community. We present relevant evidence-based literature from the 2002-2004 Severe Acute Respiratory Syndrome (SARS) outbreak. We dive into the struggle between resuming state-of-theart surgical care while maintaining social distancing and using all precautions to limit the spread of COVID19. We explore pertinent terminology, such as elective, emergent, urgent, and essential/nonessential surgery. Last, we discuss and recommend guardrails to allow the continuation of best practices and high-quality patient care while maintaining patient and staff safety. This includes reviewing the Centers for Disease Control and Prevention (CDC) recommendations, the use of traditional protective gear, screening forms and tests, new-technologic solutions, and more.

\section{Methods}

Because of the relatively recent onset of the COVID19 outbreak, the lack of available evidence-based literature and the timeliness required to present this information, a traditional systematic review and metaanalysis would have not served the purpose well.

We performed the following systematic searches as of April 17, 2020:

1. A systematic search of traditional databases, including:
a. Google Scholar
b. PubMed
c. Medline
d. Embase.

2. A systematic search of online media, including:
a. News websites
b. Facebook
c. Twitter
d. Instagram
e. Research Gate
f. COVID-19 designated sites (John-Hopkins Uni- versity Corona Virus resource center, Institute for Health Metrics and Evaluation, etc.).

Keywords included combinations of the following terms: "COVID-19," "Corona Virus," "Severe Acute Respiratory Syndrome," and "SARS" in conjunction with "Surgery," "Orthopedics" and "Elective/emergent/ urgent procedures." Articles were screened by 4 independent reviewers, and relevant key information was extracted, with an emphasis on information regarding the 2002-2004 SARS outbreak, diseasespread timelines, restrictions and orders affecting orthopedic patients and care-provider communities, recommendations to proceed with or halt various types of surgical care, and precautions that can be taken to mitigate the spread of COVID-19.

Our goal was to summarize existing recommendations and considerations so as to allow orthopedic care providers to manage orthopedic patients safely during the COVID-19 pandemic.

\section{What Can We Learn from the 2002-2004 SARS Outbreak?}

When looking into the future, we can use lessons from the past. Perhaps the only real evidence-based literature available to date is from the Severe Acute Respiratory Syndrome (SARS) outbreak in 2002-2004, caused by SARS-CoV. ${ }^{4}$ Although there are many obvious significant differences between the 2 outbreaks, this is perhaps the most recent similar experience humanity has had to endure, and we can definitely learn from it. Notably, the SARS outbreak was controlled (or it subsided) in the absence of sophisticated diagnostic tests, effective therapies or a vaccine. $^{5}$ The success of eliminating the outbreak was attributed to a multifaceted approach that included isolation of suspected cases, contact tracing, quarantine of potentially exposed individuals, provision of PPE and training for health care workers, establishment of fever clinics, and enhanced communication efforts for health care professionals, policymakers and the public. $^{5}$ Studies have shown that during the SARS outbreak, $22 \%$ of all persons affected in Hong Kong and $43 \%$ of all affected persons in Toronto were health care workers. ${ }^{6}$ Li et al. studied SARS infection among health care professionals in Beijing, China, during the 20022004 SARS outbreak. They found that among the 770 health care workers who had contact with patients who had SARS, $2.43 \%(\mathrm{~N}=18)$ were found to be infected with the virus. Importantly, no transmission was reported among hospital staff. They also reported that there was no use of negative pressure or N95 masks in their facilities and that simple protective measures and strictly enforced safety protocols were sufficient to control the in-hospital spread of SARS. ${ }^{7}$ Although Li et al. did not deem the use of N95 masks and negativepressure isolation rooms necessary, the Canadian experience reported that the following protective measures were recommended: the use of negativepressure isolation rooms, where available; N95 or higher levels of respiratory protection; gloves, gowns and eye protection; and careful hand hygiene. ${ }^{8}$ Simple PPE was also found to be effective in a systematic review performed by Jefferson et al. and published in 2007. ${ }^{9}$ Chu et al. investigated the impact of SARS on hospital performance in Taipei, Taiwan. ${ }^{10}$ They reported the average monthly number of outpatient visits at base year was 52,317 \pm 4204 . Outpatient visits 
for the year SARS emerged and 1 year and 2 years later were $55 \%, 82 \%$ and $84 \%$, respectively. Orthopedic surgery was 1 of the outpatient departments that had not recovered by the fourth year after the SARS outbreak, with an estimated $84 \%$ of outpatient visits from baseline. Schull et al. reported that the rate of elective surgery in Toronto fell by $22 \%$ and $15 \%$ during the early and late restriction periods, respectively, and by $8 \%$ in the comparison regions (Ottawa and London, Ontario). ${ }^{11}$

COVID-19 shares $70 \%-80 \%$ of its genome with SARS-CoV; both have relatively similar aerosol and surface stability are transmitted by droplets and contact with infected persons and surfaces, and both target the angiotensin-converting enzyme 2 receptor. ${ }^{12,13}$ Although the COVID-19 transmission rate seems to be much higher, the fatality rate is estimated to be less than $2.3 \%$ and perhaps much lower. In comparison, SARS-CoV and Middle East respiratory syndrome (MERS)-CoV have a reported case fatality rate of $9.6 \%$ and $35 \%$, respectively. ${ }^{12,14}$

A higher transmission rate may be related to inherent viral properties but also may, in part, be attributed to globalization, frequent air travel, and other changes in social habits that have occurred since 2004. A lower case fatality rate may also be attributed to inherent viral characteristics. Other contributing factors may include the improvement of health care services and protective equipment and other significant protective measures taken worldwide.

In summary, the 2002-2004 SARS outbreak teaches us that health care workers are at high risk and that PPE and its proper use are essential. It is of concern that the current pandemic may re-emerge next winter and that recovery of health care services may take several years or longer.

\section{Review of the National and Regional Guidelines}

By the time the World Health Organization declared COVID-19 a global pandemic, on March 11, it was apparent that the virus would place substantial strain on health care infrastructure and supplies throughout the country in the following days and weeks. ${ }^{15}$ To meet these challenges, on March 13, the American College of Surgeons put forth the first recommendations concerning elective care during this crisis. They recommended that each surgeon "thoughtfully review all scheduled elective procedures with a plan to minimize, postpone, or cancel electively scheduled operations." They also stated that this recommendation should be in place until we have passed the anticipated inflection point of virus exposure and can reliably support a rapid uptick in patients' critical-care needs. ${ }^{16}$ This statement was quickly supported by the U.S. Surgeon General and followed-up by the Centers for Medicare $\&$ Medicaid Services, which provided a tiered framework to follow when considering elective medical services. ${ }^{17,18}$ The 3 tiers, which are based upon acuity, are a guide to which procedures are appropriate to be conducted at this time and which should be postponed. In general, they recommended that surgeons limit all nonessential planned surgeries until further notice. ${ }^{17}$ Notably, initial recommendations were considered in the context of a global crisis and did not contemplate differences in local or regional disease burden.

Following these initial recommendations, most of the country's local hospital policies regarding elective surgical care have been guided by individual states' recommendations. At the time of this writing, 35 states and the District of Columbia have issued individual statements or policies pertaining to elective-care restrictions. ${ }^{19}$ However, the recommendations have been dynamic and varied, with differing definitions of elective procedures and rare references to orthopedic practices in particular. ${ }^{20}$ As such, individual institutions have been responsible for determining which orthopedic surgeries should be performed or postponed as they navigate the pandemic. Similarly, most discussions related to surgery policies initially pertained to the performance of surgery in a hospital or inpatient setting where the disease burden is greatest and resource availability is most challenged.

The many recommendations put forth raise some essential considerations as orthopedic surgeons adjust to the likely long-term impact that COVID-19 will have on surgical care. In this early period, with thousands of deaths occurring each day and health systems still under extreme stress, the decision to postpone elective procedures and allocate all resources to the crisis has been clear. However, now that conditions have seemingly started to stabilize in certain regions, surgeons must weigh the relative risks of resuming their essential elective practice while taking all possible precautions to limit COVID-19's spread. This should be performed only following the White House and CDC "Opening Up America Again" guidelines, as well as relevant federal, state and local public health guidance. ${ }^{21}$ Currently, the resumption of elective procedures is planned to proceed during Phase I of the 3-phase approach of the "Opening Up America Again" initiative.

\section{Moving Forward}

The Institute for Health Metrics and Evaluation at the University of Washington is supplying up-to-date projections of COVID-19, including deaths per day, total deaths and hospital resource use. ${ }^{22}$ The projections take into account the stay-at-home order, the closure of education facilities and nonessential services and severe travel limitations, all implemented on different dates in different states. Although the projections change with 
time, it appears that the outbreak has already peaked in many states and that many states will not sustain a deficit in hospital resources.

These projections support the motion to lift the ban, responsibly and gradually, on essential elective surgery worldwide. Lifting the constraints should not be done carelessly, and many factors should be taken into account, including the regional health care resources available and the projection of a peak in transmissions and deaths in specific geographic regions. The resumption of shoulder, knee and ankle arthroscopies (and other orthopedics procedures requiring $\leq 23$ hours of hospital stay) in Singapore can be used as an example. $^{23}$

Consistent with the need to provide additional essential services, the White House and the CDC are currently supporting the reopening of elective surgery during Phase I. This support also includes social distancing, return to work in stages, resumption of elective surgeries, and the opening of gyms, while schools and organized youth activities will remain closed.

It also proposes the formulation of region-specific plans for how to operationally resume the practice of patient care as well as how to survive financially during these turbulent times. ${ }^{24-26}$ Private practices, hospitals and health care systems (similar to other small and large businesses) are in jeopardy. With elective surgery constituting upwards of $47 \%$ of orthopedic-care spending, ${ }^{2}$ essential medical practices may default, resulting in irreparable long-term damage to public health.

Many new surgical management algorithms are circulating, and though there are some differences, most algorithms agree with the following criteria for performing essential surgery without further delay $^{21,27,28}$ :

- Locations where the outbreak has most likely peaked

- Downward trajectory of $\geq 14$ days in regional symptomatic and confirmed cases

- No shortage of resources, including:

$\circ$ Staff

- Beds

$\circ$ PPE

- Ventilators

- Patients who are healthy and asymptomatic.

- Patients ASA (American Society of Anesthesiologists) $1-2$

Patients must understand the possibility of an additional risk that is entailed by undergoing surgery during the COVID-19 outbreak and must provide written informed consent that speaks to the necessity and the risks and benefits of receiving care at this time. In addition, the surgical management algorithms should be considered in the context of hospital-based inpatient procedures on the one hand and outpatient-based procedures commonly conducted in ASCs.

Initially, ASCs are perhaps the best facilities to be used in this setting because most do not include emergency departments that typically admit elderly patients with infectious diseases and/or severe comorbidities. Moreover, most are currently vacant and have not been transitioned to receive patients with COVID-19 because in most states, there was no need to do so, to date.

We should remember that when removing these constraints, it will be critical to track any suspicion of a local surge in infections and immediately contain the local outbreak by all means, including immediate reporting to all relevant authorities.

\section{Definition of Types of Surgery}

One key consideration is the distinction between essential and nonessential surgery. Initial recommendations in mid-March in the United States were focused primarily on managing disease burden, such that the hospital system in general would not be overwhelmed in terms of available resources, including PPE, ventilators and manpower. In addition, the benefits of mitigation and social distancing were emphasized to meet that same goal. Thus, "elective surgery" was determined to be "nonessential." Subsequently, discussions ensued that related to activities that are essential, with "emergent" procedures being the most clearly defined. Interchangeably, the terms, including "urgent," were used often when referring to conditions that, if left untreated over some subsequent period of time (i.e., 46 weeks), the outcome of neglecting that condition would have a negative impact on the patient's condition. Urgent conditions would implicitly include those that are also considered emergent conditions, but arguably, there is latitude, depending on tangible and intangible considerations, to discuss what other conditions would fall into the urgent category. The 4-6 week time line was essentially based on expectations of a greater clarity related to changes in disease burden and the effects of mitigation and social distancing, with some additional insights into the timing and duration of stay-at-home orders. Notably, these same discussions have evolved to consider local demands concerning the disease burden on the hospital system rather than the global impact of the disease state on the entire hospital or medical system.

Some states have provided additional guidance. The Illinois Department of Public Health, for example, defined elective procedures as those that are preplanned by both the patient and the physician and are advantageous to the patient but are not urgent or emergent. As such, sports medicine and orthopedic procedures in many institutions during this crisis have been limited to those thought to be urgent or emergent, such as acute tendon injury, ligamentous 
tears, bucket-handle meniscus tears with a locked knee, intra-articular loose bodies causing acute symptoms, acute fracture management, dislocated joints that cannot be closed-reduced, and infected joints, among several others. Expansion of these definitions, which are relatively easy to define as emergent conditions requiring relatively immediate treatment (as a class of essential services), has included discussions related to conditions that are urgent (short of being emergent), whereby surgical intervention is required to otherwise avoid worsening the condition should surgery be delayed. Related to this is the further consideration that pain, dysfunction and loss of occupation can relate to the concept of urgency for an individual patient and, thus, essential provision of timely care may be indicated.

Other states have provided guidance concerning what to consider as essential or nonessential for the discretion of licensed providers. In Ohio, for example, nonessential surgery is defined as a procedure that can be delayed without undue risk to the current or future health of a patient. However, in areas where resources are available and health systems are adequately equipped to handle an uptick in COVID19 cases, it may be appropriate to broaden the definition of essential procedures. As an example, outpatient hip, knee and shoulder arthroscopies produce little burden on hospital resources and can be reliably performed at outpatient ambulatory surgery centers. ${ }^{23}$ In these cases, postponing care may needlessly prolong patient pain, disability and loss of occupation.

Although most would agree on what is an urgent or emergent orthopedic condition requiring surgical intervention, broadening the definition of essential surgery may be more difficult and subjective. Furthermore, practice patterns today will probably be in place for the foreseeable future and "living with COVID" will continue to guide our policies and procedures.

Our institution (Midwest Orthopaedics at Rush University Medical Center) has implemented the following definitions of urgent surgery to be performed in a limited capacity in the ASC system that are not necessarily considered emergent but, rather, urgent and, thus, essential based on relevant clinical parameters. Ultimately, decisions may be multifactorial and made with board oversight and comprehensive documentation and may depend on local resource demands, downward changes in the disease burden and federal and local policies. ${ }^{29}$ These factors might include:

1. Conditions that without treatment could result in compromised outcomes

2. A condition that has failed to respond to nonsurgical care
Table 1. Recommended Precautions and Actions: The Patient

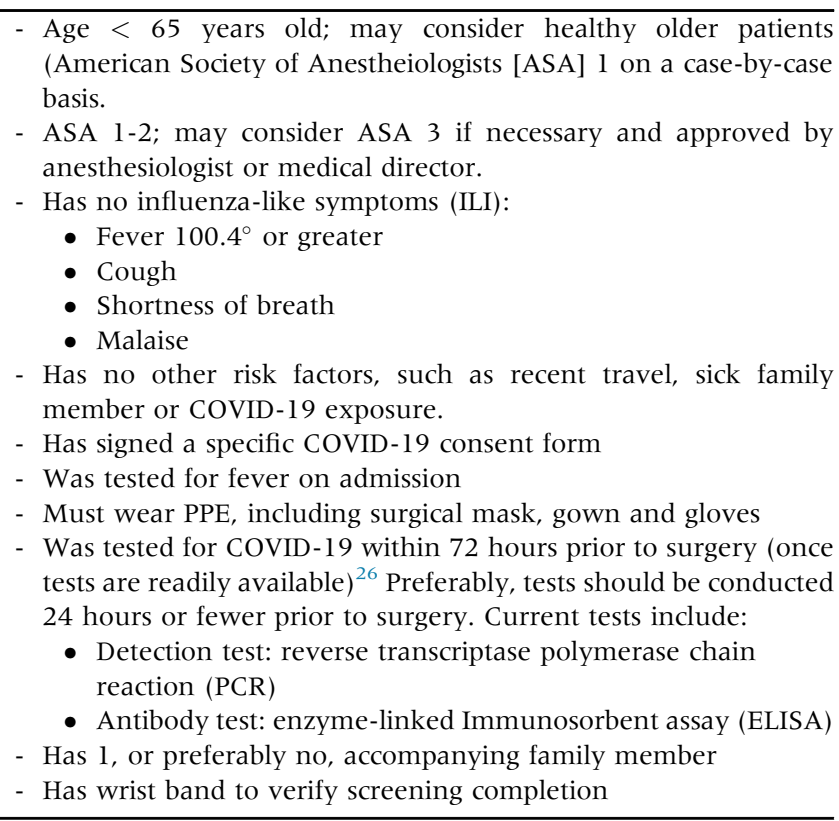

3. Neurologic deficits and/or progressive neurologic deterioration

4. Conditions with intolerable pain, especially when narcotics are required

5. Functional losses precluding return to activities, including activities of daily living

6. Conditions resulting in significant financial hardship.

\section{Protective Measures}

In contemplating living with COVID-19, we must assume that the protective measures we implement today may remain with us for years to come. Jefferson et al. demonstrated that routine long-term implementation of PPE to interrupt or reduce the spread of respiratory viruses is effective, and many simple and low-cost interventions could be useful in reducing their spread. ${ }^{9}$

Table 2. Recommended Precautions and Actions: The Staff

- Hand hygiene is key.

- Staff should keep social distancing (minimum 6-foot distance when possible) and use personal protective equipment (gloves, gown, surgical mask, and goggles).

- Intubation should be performed with only the necessary staff in the operating room, wearing N95 masks and eye protection.

- Delays between room re-entrance by necessary staff and between cases.

- Minimize staffing as much as possible.

- Test all staff for COVID-19 on arrival or before (once tests are readily available).

- Wear wrist band to verify screening completion.

- Staff should be trained in protecting themselves and patients.

- Provide supportive measures to address staff fatigue and emotional distress. 
Table 3. Recommended Precautions and Actions: Facility and Region

Each geographic region and facilities within it must assess the availability of the following and proceed accordingly if a shortage occurs or is expected ${ }^{32}$ :

- Personal protective equipment supply

- Staffing

- Beds (specifically, intensive care unit beds)

- Ventilators

- Medications, anesthetics and all surgical supplies

Facilities should implement the following:

- A single exit and a single entrance

- Elevator management to minimize crowding of patients and staff

- Operating/procedural rooms must meet engineering and Facility Guideline Institute standards for air exchanges.

- Protocols for managing and isolating patients and staff suspected of or confirmed to have COVID-19 infection

- Case prioritization strategy is set in place.

- Data should be collected as proposed by the American College of Surgeons in order to reassess policies and procedures frequently. Other regional considerations include ${ }^{26}$ :

- A sustained reduction in COVID-19 cases for $\geq 14$ days

- Access for COVID-19 testing

- Availability of active monitoring of confirmed or suspected cases and their contacts

Tables 1-5 list precautions and actions that may limit transmission, protect patients and health care providers and allow the resumption of essential surgical services. $^{30-32}$

\section{Limitations}

A significant limitation of all studies investigating aspects of the COVID-19 pandemic is the lack of evidence-based, trustworthy resources. This study is unique in its attempt to analyze studies performed following the 2002-2004 SARS outbreak, but recent literature concerning the COVID-19 pandemic is mostly Level $\mathrm{V}$ evidence. Moreover, because of the rapidly unfolding reality we are facing, this article, like many others pertaining to the COVID-19 pandemic, may not be relevant within a few weeks or even a few days.

\section{Summary}

"First, do no harm" is embedded in our core as health care providers in addition to the privilege to help those in need. We must limit all unnecessary activities to

Table 4. Recommended Precautions and Actions: Surgery

\footnotetext{
- Same-day preoperative admissions are preferred (rather than a day before).

- Updated preoperative checklists with questions pertaining to COVID-19

- Surgical times should be kept short.

- Limited number of operations per operation room block

- Limit operating room traffic

- Disinfect the operating room strictly.

- Additional room turnover delay as necessary

- Expedited postoperative recovery and discharge procedures
}

Table 5. Recommended Precautions and Actions: Postoperative Management

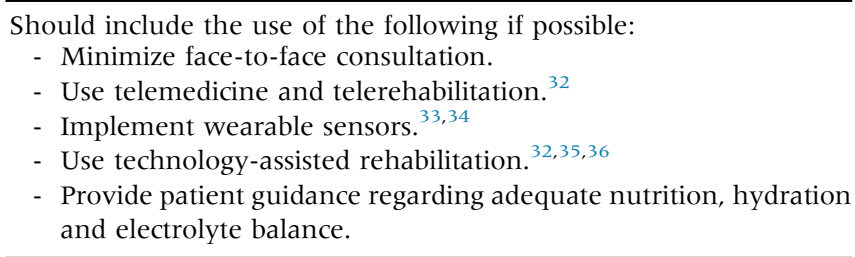

mitigate the harmful effects of this pandemic. At the same time, we must remember that in our profession, failure to act and provide essential care can be irreversibly detrimental to patients' health and quality of life. Whether the COVID-19 outbreak is close to an end, whether the outbreak will reemerge next winter, and whether another contagious pathogen will appear are unknown. However, it is clear that we must adapt and find ways to resume our lives and provide high-quality medical and surgical care while using the best protective measures available to protect our patients and staff.

\section{References}

1. Holshue ML, DeBolt C, Lindquist S, Lofy KH, Wiesman J, Bruce H, et al. First case of 2019 novel Coronavirus in the United States. N Engl J Med 2020;382:929-936.

2. Planned knee and hip replacement surgeries are on the rise in the U.S.: Blue Cross Blue Shield, 2019.

3. Gawande A. Keeping the coronavirus from infecting health-care workers. The New Yorker. March 21, 2020. https://www.newyorker.com/news/news-desk/keepingthe-coronavirus-from-infecting-health-care-workers.

4. Lee N, Hui D, Wu A, Chan P, Cameron P, Joynt GM, et al. A major outbreak of severe acute respiratory syndrome in Hong Kong. N Engl J Med 2003;348:1986-1994.

5. Hughes JM. The SARS response-building and assessing an evidence-based approach to future global microbial threats. JAMA 2003;290:3251-3253.

6. Naylor CD, Chantler C, Griffiths S. Learning from SARS in Hong Kong and Toronto. JAMA 2004;291:2483-2487.

7. Li L, Cheng S, Gu J. SARS infection among health care workers in Beijing, China. JAMA 2003;290:2662-2663.

8. Control CfD, Prevention. Cluster of severe acute respiratory syndrome cases among protected health-care workers: Toronto, Canada, April 2003. MMWR 2003;52: 433.

9. Jefferson T, Foxlee R, Del Mar C, Dooley L, Ferroni E, Hewak B, et al. Physical interventions to interrupt or reduce the spread of respiratory viruses: Systematic review. BMJ 2008;336:77-80.

10. Chu D, Chen R-C, Ku C-Y, Chou P. The impact of SARS on hospital performance. BMC Health Serv Res 2008;8:228.

11. Schull MJ, Stukel TA, Vermeulen MJ, Zwarenstein M, Alter DA, Manuel DG, et al. Effect of widespread restrictions on the use of hospital services during an outbreak of severe acute respiratory syndrome. CMAJ 2007;176:1827-1832. 
12. Ceccarelli M, Berretta M, Rullo EV, Nunnari G, Cacopardo B. Differences and similarities between severe acute respiratory syndrome (SARS)-Coronavirus (CoV) and SARS-CoV-2: Would a rose by another name smell as sweet? Eur Rev Med Pharmacol Sci 2020;24:2781-2783.

13. van Doremalen $\mathrm{N}$, Bushmaker $\mathrm{T}$, Morris $\mathrm{DH}$, Holbrook MG, Gamble A, Williamson BN, et al. Aerosol and surface stability of SARS-CoV-2 as compared with SARS-CoV-1. N Engl J Med 2020;382:1564-1567.

14. Arabi YM, Arifi AA, Balkhy HH, Najm H, Aldawood AS, Ghabashi A, et al. Clinical course and outcomes of critically ill patients with Middle East respiratory syndrome coronavirus infection. Ann Intern Med 2014;160:389-397.

15. WHO Director-General's opening remarks at the media briefing on COVID-19. 11 March 2020. World Health Organization, 2020.

16. COVID-19: Recommendations for management of elective surgical procedures. Vol. 2020: American College of Surgeons, 2020.

17. CMS Releases recommendations on adult elective surgeries, non-essential medical, surgical, and dental procedures during COVID-19. Response: Center for Medicare \& Medicaid Services, 2020.

18. Luthi S. Surgeon General advises hospitals to cancel elective surgeries. Vol. 2020. Politico2020.

19. ASCA. State guidance on elective surgeries, Vol. 2020. Ambulatory Surgery Center Association, 2020.

20. Sarac NJ, Sarac BA, Schoenbrunner AR, Janis JE, Harrison RK, Phieffer LS, et al. A review of state guidelines for elective orthopaedic procedures during the COVID-19 Outbreak [published online April 13, 2020]. J Bone Joint Surg Am. doi:10.2106/JBJS.20.00510.

21. CDC, White House. Opening Up America Again guidelines: 2020.

22. The Institute for Health Metrics and Evaluation (IHME), University of Washington. COVID-19 Projections. 2020.

23. Liang ZC, Wang W, Murphy D, Hui JHP. Novel coronavirus and orthopaedic surgery: Early experiences from Singapore [published online March 20, 2020]. J Bone Joing Surg Am. doi:10.2106/JBJS.20.00236.

24. Vaccaro AR, Getz CL, Cohen BE, Cole BJ, Donnally CJ III. Practice management during the COVID-19 Pandemic [published online April 13, 2020]. J Am Acad Orthop Surg. doi:10.5435/JAAOS-D-20-00379.

25. Gilat R, Cole BJ. COVID-19, medicine, and sports [published online April 11, 2020]. Arthrosc Sports Med Rehabil. doi:10.1016/j.asmr.2020.04.003.

26. American Academy of Orthopaedic Surgeons. Navigating the COVID-19 pandemic. 2020.

27. Schmidt T. AAOS: Elective surgical procedures. 2020.

28. Stahel PF. How to risk-stratify elective surgery during the COVID-19 pandemic? New York: Springer, 2020.

29. CMS. Opening Up America Again. Centers for Medicare e Medicaid Services (CMS) recommendations re-opening facilities to provide non-emergent non-COVID-19 Healthcare: Phase I. 2020.

30. Brücher BL, Nigri G, Tinelli A, Lapeña JF, Espin-Basany E, Macri P, et al. COVID-19: Pandemic surgery guidance. 4open 2020;3:1.

31. Awad ME, Rumley JC, Vazquez JA, Devine JG. Perioperative considerations in urgent surgical care of suspected and confirmed COVID-19 orthopedic patients: Operating rooms protocols and recommendations in the Current COVID-19 pandemic [published online April 10, 2020]. J Am Acad Orthop Surg. doi:10.5435/JAAOSD-20-00227.

32. Surgeons ACo. Joint Statement: Roadmap for Resuming Elective Surgery after COVID-19 Pandemic. 2020.

33. Small SR, Bullock GS, Khalid S, Barker K, Trivella M, Price AJ. Current clinical utilisation of wearable motion sensors for the assessment of outcome following knee arthroplasty: a scoping review. BMJ Open 2019;9.

34. Chiang C-Y, Chen K-H, Liu K-C, Hsu SJ- P, Chan C-T. Data collection and analysis using wearable sensors for monitoring knee range of motion after total knee arthroplasty. Sensors 2017;17:418.

35. Koo K-i, Park DK, Youm YS, Do Cho S, Hwang CH. Enhanced reality showing long-lasting analgesia after total knee arthroplasty: Prospective, randomized clinical trial. Sci Rep 2018;8:1-9.

36. Chen M, Li P, Lin F. Influence of structured telephone follow-up on patient compliance with rehabilitation after total knee arthroplasty. Patient Prefer Adherence 2016; 10:257. 